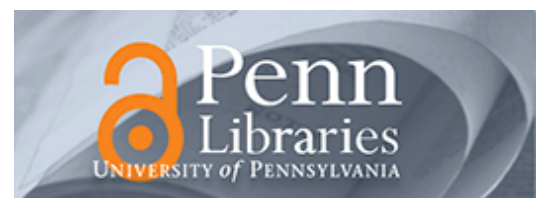

University of Pennsylvania

ScholarlyCommons

May 1995

\title{
Control of forward velocity for a simplified planar hopping robot
}

\author{
William J. Schwind \\ University of Michigan \\ Daniel E. Koditschek \\ University of Pennsylvania, kod@seas.upenn.edu
}

Follow this and additional works at: https://repository.upenn.edu/ese_papers

\section{Recommended Citation}

William J. Schwind and Daniel E. Koditschek, "Control of forward velocity for a simplified planar hopping robot", . May 1995.

Copyright 1995 IEEE. Reprinted from Proceedings of the IEEE International Conference on Robotics and Automation, Volume 1, 1995, pages 691-696.

This material is posted here with permission of the IEEE. Such permission of the IEEE does not in any way imply IEEE endorsement of any of the University of Pennsylvania's products or services. Internal or personal use of this material is permitted. However, permission to reprint/republish this material for advertising or promotional purposes or for creating new collective works for resale or redistribution must be obtained from the IEEE by writing to pubs-permissions@ieee.org. By choosing to view this document, you agree to all provisions of the copyright laws protecting it.

NOTE: At the time of publication, author Daniel Koditschek was affiliated with the University of Michigan. Currently, he is a faculty member in the Department of Electrical and Systems Engineering at the University of Pennsylvania.

This paper is posted at ScholarlyCommons. https://repository.upenn.edu/ese_papers/377

For more information, please contact repository@pobox.upenn.edu. 


\title{
Control of forward velocity for a simplified planar hopping robot
}

\author{
Abstract \\ A simplified lossless model of the Raibert planar hopper is introduced for the purpose of analytically \\ studying the control of forward velocity. A closed-form return map describing the robot's state at the next \\ hop as a function of that at the current hop is derived. The Raibert forward velocity controller is \\ introduced and the fixed points of the closed loop system are characterized as well as the stability of \\ these points. A new control law inspired by this analysis is introduced and compared with the Raibert \\ control law.
}

\section{Comments}

Copyright 1995 IEEE. Reprinted from Proceedings of the IEEE International Conference on Robotics and Automation, Volume 1, 1995, pages 691-696.

This material is posted here with permission of the IEEE. Such permission of the IEEE does not in any way imply IEEE endorsement of any of the University of Pennsylvania's products or services. Internal or personal use of this material is permitted. However, permission to reprint/republish this material for advertising or promotional purposes or for creating new collective works for resale or redistribution must be obtained from the IEEE by writing to pubs-permissions@ieee.org. By choosing to view this document, you agree to all provisions of the copyright laws protecting it.

NOTE: At the time of publication, author Daniel Koditschek was affiliated with the University of Michigan. Currently, he is a faculty member in the Department of Electrical and Systems Engineering at the University of Pennsylvania. 


\title{
Control of Forward Velocity for a Simplified Planar Hopping Robot
}

\author{
William J. Schwind* and Daniel E. Koditschek ${ }^{\dagger}$ \\ Department of Electrical Engineering and Computer Science \\ The University of Michigan \\ Ann Arbor, MI 48109-2122, USA
}

\begin{abstract}
A simplified lossless model of the Raibert planar hopper is introduced for the purpose of analytically studying the control of forward velocity. A closed-form return map describing the robot's state at the next hop as a function of that at the current hop is derived. The Raibert forward velocity controller is introduced and the fixed points of the closed loop system are characterized as well as the stability of these points. A new control law inspired by this analysis is introduced and compared with the Raibert control law.
\end{abstract}

\section{Introduction}

This paper concerns the behavior of a planar hopping robot where the only control exerted is the placement of the leg at touchdown. This work is motivated by the dramatic success achieved by Marc Raibert in implementing simple control strategies to control physical hopping robots [5].

Raibert and his students built and studied hopping robots as a means to understand dynamic legged locomotion [5]. The first hopper was constrained to move in the plane. It had a pneumatic cylinder for its leg and hence acted as a springy inverted pendulum while on the ground. For this planar hopper, Raibert introduced simple control laws which successfully regulated vertical hopping height, forward velocity and body attitude. The understanding gained from the planar hopper was exploited to successfully implement three dimensional hoppers, biped hoppers and quadraped hoppers.

Raibert sees the tasks of regulating hopping height, forward velocity and body attitude as three separate control problems. Each controller is designed assuming that the behavior to be controlled is decoupled from the other behaviors. The experimental success of such

*Supported in part by a National Science Foundation Graduate Research Fellowship

tSupported in part by the National Science Foundation under grant IRI-9123266 a controller design validates the decoupling assumption. Previous work has endeavored to understand the control of vertical hopping height [3] [8] [7] and how it is influenced by the addition of the forward running dynamics [4]. In this paper, we restrict attention to the control of forward velocity. Specifically, this is accomplished by deriving a return map for a simplifed version of the Raibert planar hopper, wherein we assume no energy losses and constant angular momentum during stance. Control is exerted solely in the forward placement of the leg at touchdown. We introduce a feedback control law derived from Raibert's reported procedure, characterize the set of fixed points for the resulting closed loop system, and initiate a study of the stability properties of these fixed points by means of local analysis and numerical simulation.

\subsection{Overview of the Paper}

This paper begins by introducing in section 2 a simplified model of the planar hopper. A return map describing the robot's state at next hop as a function of its state at the current hop is derived in section 3 and represents a central contribution of this paper. Once the return map is derived, control is introduced in section 4 and a study the fixed points of the resulting closed loop map and their stability properties is undertaken.

\section{A Simplified Model of the Two Degree of Freedom Hopper}

Figure 1 shows the simplified planar hopper. We will assume that the leg is massless and that the body is a unit point mass. Modelling the hopper in this way removes any need to consider control of body attitude, since the body has no rotational inerti: about the leg. The leg is a pneumatic cylinder, which acts simultaneously as a prismatic joint and an energy storage mechanism where the force is inversely proportional to leg length. 


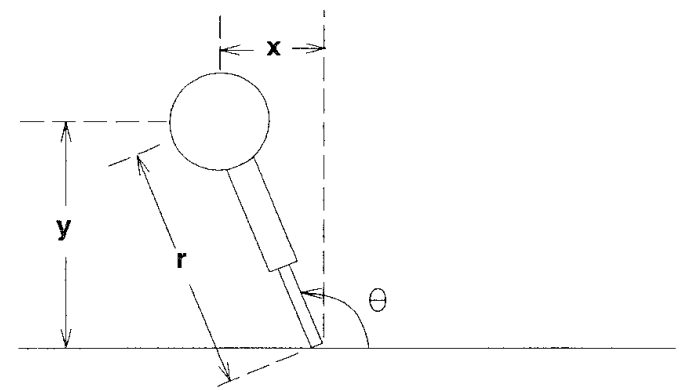

Figure 1: The simplified planar hopper

\subsection{Operation of the Hopper}

The hopping cycle consists of two primary phases: the stance phase, when the foot is on the ground, and the flight phase, when the robot is airborne [5]. The stance phase can be decomposed into three sub-phases: compression, thrust and decompression. Four important events must occur during one hopping cycle: Touchdown, the moment the foot makes contact with the ground; Bottom, the moment during stance when the robot reaches maximal compression and the radial velocity changes from inward to outward; Lift-Off, the moment the foot loses contact with the ground; Apex, the moment in the flight phase where the robot has maximum amplitude and vertical motion changes from upward to downward.

\subsection{Control of the Hopper}

The control task for the simplified hopper is first, to regulate about a certain apex height; second, to regulate about a certain forward velocity. Raibert attempts to achieve these goals by treating hopping height and forward speed as separate control problems. This decoupling of the control relies on a presumed weak coupling between the motions. He summarizes the control tasks as follows [5]:

1) Excite hopping motions and regulate their amplitude by specifying the thrust to be delivered by the leg on each hop.

2) Stabilize the machine's forward speed by extending the foot forward to a position that will provide the needed acceleration during stance.

The second author has previously studied Raibert's solution to 1) in isolation [3]. We now undertake the study of 2) in isolation.

\subsubsection{Control of Forward Speed}

Since forward speed is constant during flight, any acceleration must occur during stance phase. In light of this, Raibert observes that the angle of the leg at touchdown has a powerful effect on the accelerations that occur during stance.

Raibert's strategy for selecting the touchdown angle is based upon his observation that for each forward velocity there is a unique touchdown angle that results in zero net forward acceleration. This angle will be called the neutral angle and the foot postion corresponding to this angle will be called the neutral point. When the foot is placed on the neutral point, the motion of the body during the stance phase is symmetric resulting in zero net forward acceleration. Conversely, displacing the foot from the neutral point generates either net forward accelerations or decelerations.

Raibert's approach was to use simple approximations to estimate the location of the neutral point and choose a forward position for the foot that effects the desired net forward acceleration. The control law he implemented took the form

$$
x_{f}=\frac{\dot{x} T_{s}}{2}+k_{\dot{x}}\left(\dot{x}-\dot{x_{d}}\right)
$$

Where,

$x_{f} \quad$ is the forward displacement of the foot with respect to the center of mass

$T_{s}$ is the duration of the stance phase

$\dot{x} \quad$ is the forward speed

$\dot{x}_{d} \quad$ is the desired forward speed

$k_{\dot{x}} \quad$ is a feedback gain

Here, $\frac{\dot{x} T_{s}}{2}$ serves as an approximation for the neutral point and $k_{\dot{x}}\left(\dot{x}-\dot{x_{d}}\right)$ serves as the displacement of the foot from the neutral point to achieve the necessary forward accelerations.

We will consider the planar hopper with forward velocity control only. Since there is no thrust, we have no means of either injecting energy into the system or changing the hopper's spring constant during stance. As a consequence, we will assume there are no dissipative losses throughout the hopping cycle. This assumption is reasonable since the energy added by thrust during each stance is meant to compensate for the losses that take place throughout the cycle. We follow Raibert's ideas as detailed above and let the forward position of the leg at touchdown, or equivalently the touchdown angle, $\theta=\theta_{t d}$ be the control input.

\subsection{Dynamics of the Simplified Hopper}

We will use Cartesian coordinates, $b:=[x, y]^{T}$ and polar coordinates $q:=[r, \theta]^{T}$ as depicted in Figure 1. The stance phase begins at touchdown, where the leg is fully extended, $r=r_{t d}$. The leg cylinder pressure is fixed to be the same at each touchdown, yielding a spring constant of $\omega_{0}^{2}$ throughout the stance phase. The length of the pneumatic spring during stance is $\left(r-r_{p}\right)$, where $r_{p}$ is the length of 
the piston. The leg compresses until it reaches the botton state and then begins to decompress. The hopper lifts off when the leg is again fully extended, $r=r_{l o}=r_{t d}$. Under the assumptions discussed above and assuming a spring law with potential $U(r)$, we find that the potential energy during stance is given by $V=g r \sin \theta+U(r)$ and the kinetic energy during stance is given by $T=\frac{1}{2}\left[\dot{r}^{2}+(r \dot{\theta})^{2}\right]$. Defining the Lagrangian function, $L(q, \dot{q}, t)=T-V$ and applying the Lagrangian operator yields the following stance dynamics:

$$
\ddot{\boldsymbol{q}}=\left[\begin{array}{l}
\ddot{r} \\
\ddot{\theta}
\end{array}\right]=\left[\begin{array}{c}
r \dot{\theta}^{2}-D U(r)-g \sin \theta \\
-\frac{2 \dot{r} \dot{\theta}+g \cos \theta}{r}
\end{array}\right]
$$

During the flight phase, the robot acts as a projectile under the force of gravity. Hence the flight dynamics are governed by

$$
\ddot{b}=\left[\begin{array}{l}
\ddot{x} \\
\ddot{y}
\end{array}\right]=\left[\begin{array}{c}
0 \\
-g
\end{array}\right]
$$

A two degree of freedom mechanical system can be completely described by its planar positions and velocities, thus the hopper's dynamics reside in a four dimensional phase space. By selecting a Poincaré section and studying the systems evolution on this section, we can reduce by one the dimension of the problem to be studied. Furthermore, we will see that after $\theta_{t d}$ is selected the system reduces to two dimensions.

\section{The Apex Return Map}

While various means could be used to define a Poincaré section, it is advantageous to select the apex event. ${ }^{1}$ An apex event occurs during the flight phase when the hopper reaches its maximum altitude and as a result can be defined as the subspace $\dot{\bar{y}} \equiv 0$. As a matter of notation, apex coordinates will always be denoted by an overbar. Because the hopping cycle can be thought of as descending from apex to touchdown, progressing from touchdown to liftoff, and ascending from liftoff to the new apex, the apex return map $\mathcal{R}:[\overline{\boldsymbol{b}}(k), \dot{\overline{\boldsymbol{b}}}(k)]^{T} \mapsto[\overline{\boldsymbol{b}}(k+1), \dot{\overline{\boldsymbol{b}}}(k+1)]^{T}$ can be considered as the following composition:

$$
\mathcal{R}=\mathcal{F}_{a} \circ \mathcal{S} \circ \mathcal{F}_{d}
$$

Where, $\mathcal{F}_{d}$ is the descent flight map, $\mathcal{S}$ is the stance map, and $\mathcal{F}_{a}$ is the ascent flight map.

\footnotetext{
${ }^{1}$ Only the current control input appears in the resulting return map. In contrast, both the current and previous control inputs enter the return map of the hopper in the other coordinate systems.
}

\subsection{A Simplification of the Stance Dy- namics}

Because the flight dynamics are affine in state, the bulk of the work in deriving the return map lies in integrating the stance dynamics. As is often the case for nonlinear differential equations, the stance dynamics shown above (1) do not admit closed form integration. Difficulties arise due to the presence of the gravitational terms and the choice of the spring law. Thus, as in [4] we will assume that the spring force is the dominant radial force during stance and that the robot's angular momentum is constant during stance. This allows us to ignore gravity in both the the radial and angular equations of stance motion. While simulations suggest these assumptions are reasonable, more systematic studies are being undertaken to identify the regimes where these assumptions hold.

\subsection{The Resulting Form of the Return Map}

Having found the maps for the stance and flight phases in [6], we can now complete the expression for the return map. While $\mathcal{R}$ is technically a map from $\mathbb{R}^{4}$ to itself, we have reduced its order by one by restricting the state to the Poincaré section defined by $\dot{\bar{y}}=0$. Moreover, the resulting three dimensional map is a function of $\left(\bar{y}, \dot{x}, \theta_{t d}\right)$. Since $\theta_{t d}$ is the sole horizontal velocity control input according to Raibert's design, we observe that the problem reduces to the two dimensional return map given by

$$
\begin{aligned}
& f\left(z, \theta_{t d}\right)= \\
& {\left[\begin{array}{c}
\frac{1}{2 g}\left(\dot{\bar{x}} \sin \gamma+\left[2 g\left(\bar{y}-r_{t d} \sin \theta_{t d}\right)\right]^{\frac{1}{2}} \cos \gamma\right)^{2}+r_{t d} \sin \left(\gamma-\theta_{t d}\right) \\
-\dot{\bar{x}} \cos \gamma+\left[2 g\left(\bar{y}-r_{t d} \sin \theta_{t d}\right)\right]^{\frac{1}{2}} \sin \gamma
\end{array}\right]}
\end{aligned}
$$

where $z=[\bar{y}, \dot{\bar{x}}]^{T}$ and $\gamma=\theta_{t d}+\theta_{l o}=2\left(\theta_{t d}-\Delta \theta_{b}\right)$. In the previous expression, $\Delta \theta_{b}$ represents the change in angle from touchdown to bottom; it is obtained by integrating the stance dynamics and therefore depends strongly upon the form of $U(r)[6]$.

In the ensuing analysis of the fixed points and their stability, we will find it helpful to decompose the return map into the following form:

$$
f\left(z, \theta_{t d}\right)=t_{\gamma-\theta_{t d}} \circ s \circ r_{\gamma} \circ s^{-1} \circ t_{-\theta_{t d}}(z)
$$

Where,

$$
\begin{array}{cc}
s(x)=\left[\begin{array}{c}
\left(k_{o} x_{2}\right)^{2} \\
x_{1}
\end{array}\right] ; & s^{-1}(x)=\left[\begin{array}{c}
x_{2} \\
\frac{1}{k_{o}} \sqrt{x_{1}}
\end{array}\right] \\
r_{\gamma}(x)=-\left[\begin{array}{cc}
\cos \gamma & -\sin \gamma \\
\sin \gamma & \cos \gamma
\end{array}\right] \boldsymbol{x} ; & t_{\alpha}(x)=x+\left[\begin{array}{c}
r_{t d} \sin \alpha \\
0
\end{array}\right] .
\end{array}
$$


Armed with this decomposition, we will move to consider the equilibria and stability properties of this system.

\section{Fixed Point and Stability Analysis}

Having simplified the hopper's fourth order continuous dynamics to arrive at the second order discrete dynamical control system, $f\left(\boldsymbol{z}, \theta_{t d}\right)$, we now introduce a control law $\theta_{t d}=u(z)$ in order to arrive at the closed loop control system, $f_{u}(\boldsymbol{z})=f(z, u(z))$.

\subsection{Fixed Points}

A fixed point $z^{*}$ of the hopping behavior must satisfy the relation $f_{u}\left(z^{*}\right)=t_{\left(\gamma\left(z^{*}\right)-u\left(z^{*}\right)\right)} \circ s \circ r_{\gamma\left(z^{*}\right)} \circ s^{-1} \circ$ $t_{-u\left(z^{*}\right)}\left(z^{*}\right)=z^{*}$. Let $\mathcal{F P}=\left\{z \in \mathbb{R}^{2} \mid f_{u}(z)=z\right\}$. Notice for $\gamma\left(z^{*}\right)=\pi$, we find $\left.f_{u}\right|_{\left(\gamma\left(z^{*}\right)=\pi\right)}=t_{u\left(z^{*}\right)} 0$ $s \circ I \circ s^{-1} \circ t_{-u\left(z^{*}\right)}=i d$, the identity mapping. ${ }^{2}$ Hence we find a sufficent condition for $z \in \mathcal{F P}$ is that $z \in \Gamma_{\pi}$, where $\Gamma_{\pi}=\left\{z \in \mathbb{R}^{2} \mid \gamma(z)=\pi\right\}$. Moreover, it can be shown that $z \in \Gamma_{\pi}$ is a necessary condition for $z \in \mathcal{F P}$ and hence $\Gamma_{\pi}=\mathcal{F} \mathcal{P}[6]$. In general, $\Gamma_{\pi}$ will be a curve in the $z$ plane. To illustrate this, see Figure 2 which shows the fixed point curve for the closed loop system with the control law of section 4.2 .2 introduced.

The physical significance of the condition $\gamma=\pi$ is that $\theta_{t d}$ and $\theta_{l o}$ are symmetric about $\frac{\pi}{2}$. This $\theta$ symmetry in addition to the stance symmetry $\left(r_{10}=\right.$ $r_{t d}, \dot{r}_{l o}=-\dot{r}_{t d}$ and $\left.\dot{\theta}_{l o}=\dot{\theta}_{t d}\right)$ shown in [6] yields a complete symmetry between the touchdown and liftoff conditions. This symmetry of $\theta$ about $\frac{\pi}{2}$ is exactly the relationship Raibert tries to achieve to regulate forward speed.

\subsection{Stability}

Having derived the relation which specifies the set of fixed points of $f_{u}$, we would like to characterize the stability properties of the elements of this set. Since in general we are not only concerned with the stability of equilbria, but their domain of attraction as well, this paper will provide an analysis of the local stability properties of the fixed points and use simulations to give a feel for the domain of attraction of the set of fixed points.

To study the local stability properties, we will find the eigenvalues of the Jacobian of $f_{u}$ evaluated at the fixed point condition, which is shown in [6] to be

\footnotetext{
${ }^{2}$ Technically, $\gamma\left(z^{*}\right)=(2 n+1) \pi$ is the fixed point condition. However, since all these cases coincide on our state space - topologically equivalent to the cylinder - it is unnecessary to carry along the extra $(2 n+1)$. Moreover, since $\gamma=\theta_{t d}+\theta_{l o}$, both of which lie in $[0, \pi]$, the physically meaningful set correpsonds to $n=0$.
}

$$
\begin{aligned}
& D_{\boldsymbol{z}} f_{u}\left(\boldsymbol{z}^{*}\right)=I+ \\
& {\left[\begin{array}{c}
2 k_{0} \dot{\bar{x}}^{*}\left[\bar{y}^{*}-r_{t d} \sin u\left(z^{*}\right)\right]^{\frac{1}{2}}-r_{t d} \cos u\left(z^{*}\right) \\
-\frac{1}{k_{0}}\left[\bar{y}^{*}-r_{t d} \sin u\left(z^{*}\right)\right]^{\frac{1}{2}}
\end{array}\right] D_{z \gamma}}
\end{aligned}
$$

For simplicity, we will refer to the outer product on the right hand side of the above equation as $a b^{T}$. The eigenvalues of $D_{z} f_{u}\left(z^{*}\right)$ are given by $\lambda_{1}=1$ and $\lambda_{2}=1+a^{T} b$. The unity eigenvalue arises independently of both the structure of the control input and the assumption of the specific spring law, $D U(r)$. In contrast, the second eigenvalue depends strongly on both the controller used and the spring law assumed. Since we are only exerting control for the forward velocity, it comes as no surprise that we see a unity eigenvalue at the fixed point in any case. It is expected that even if a fixed point is attracting in the $\overline{\bar{x}}$ direction, it is at best marginally stable in the $\bar{y}$ direction.

Let $\mathcal{F P} \mathcal{P}_{S}$ be the subset of $\Gamma_{\pi}$ given by $\mathcal{F} \mathcal{P}_{S}=$ $\left\{z \mid-2<a^{T} b(z)<0\right\}$. Then for all $\boldsymbol{z} \in \mathcal{F} \mathcal{P}_{S}$ the Jacobian evaluated at $\boldsymbol{z}$ has one stable eigenvalue and one unity eigenvalue. Because we are looking at the Jacobian of a nonlinear system, this test is inconclusive and we cannot immediately make any claims regarding the stability of these fixed points.

In an attempt to understand the stability of the points in $\mathcal{F} \mathcal{P}_{\mathcal{S}}$, we can observe that the center manifold is identically the set of fixed points. This is easily verified, since the center eigenspace, i.e. that corresponding to the unity eigenvalue, is orthogonal to $D_{z} \gamma$ and hence tangent to the fixed point curve [1]. Moreover, locally for points in $\mathcal{F} \mathcal{P}_{S}$ the transverse dynamics are converging towards the center manifold [6].

The attracting portion, $\mathcal{F} \mathcal{P}_{S}$, of the fixed point curve is bounded by the condition $-2<a^{T} b<0$. Since $a^{T} b$ is strongly dependent upon the choice of the spring law, $D U(r)$, and the control input, $u(z)$, we must now choose both.

\subsubsection{A Particular Spring Law}

While the structure of the return map is shown in section 3.2 , the expression for $\Delta \theta_{b}$ and hence that for $\gamma$ is left unspecified. This work is completed in [6], where an integral expression for $\Delta \theta_{b}$ is derived. The analytical tractibility of that integral depends greatly on the choice of the spring law $D U(r)$. A $\frac{1}{r^{3}}$ spring law is introduced and shown to result in a closed-form expression for $\Delta \theta_{b}$, which in turn allows us to completely specify a closed-form return map for the simplified planar hopper. The validity of the above assumption is demonstrated in [6] by showing that the force profiles for the $\frac{1}{r^{3}}$ spring law and the commonly used $\frac{1}{r-r_{p}}$ spring law, as well as the phase portraits of the hopper corresponding to these spring laws are almost identical. 


\subsubsection{The Raibert Controller}

The above analysis was undertaken independently of the controller structure. That is, any control law we choose could be substituted into the $a^{T} b$ expression and the stability of a selected fixed point, $z^{*}$ could be studied by calculating the value of $a^{T} b\left(z^{*}\right)$. In this section, we will actually look at a control law motivated by and bearing strong resemblance to the Raibert controller. Figure 2 shows the set of stable fixed points, $\mathcal{F} \mathcal{P}_{S}$, arising from the use of such a controller.

The Raibert controller for forward speed is discussed at length in section 2.2.1. Because of the analytical complexities of even the simplified stance dynamics, we have no closed form expressions for the time of stance. Hence, the approximation for the $x_{f}^{*}$ corresponding to the neutral point is given by $r_{t d} \cos \theta^{*}$, where $\theta^{*}$ is computed to be the touchdown angle which results in a fixed point with $\dot{\bar{x}}=\dot{x}^{*}$. The error term is identical to that of Raibert's. Putting this all together, the resulting control law is

$$
u(z)=\theta_{t d}(k+1)=a \cos \left[\frac{r_{t d} \cos \theta^{*}+k\left(\dot{x}^{*}-\dot{x}\right)}{r_{t d}}\right] .
$$

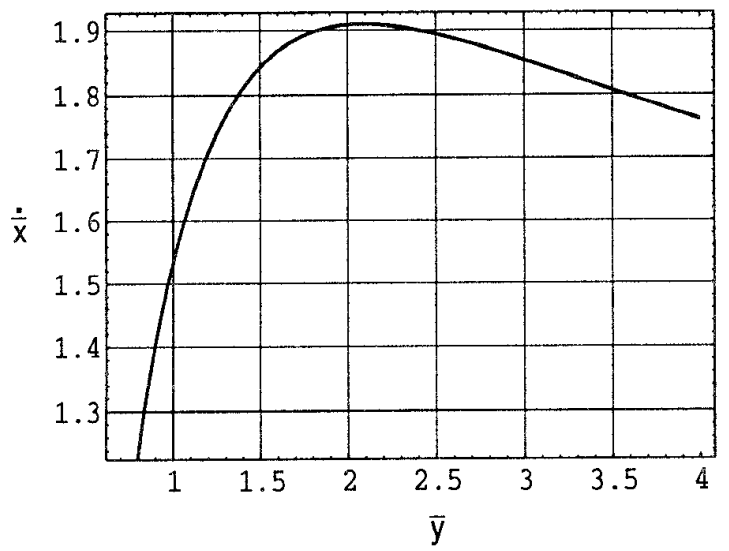

Figure 2: The set of fixed points of $f_{u}$ arising from the use of a Raibert controller with $\dot{x}^{*}=1.8$ on a simplified hopper with $r_{t d}=.7, \omega_{o}^{2}=16$.

The closed loop return map $f_{u}$ with the control law described above has been simulated using dstool, a dynamical systems simulation package designed by Guckenheimer and his students at Cornell University [2]. Using this package we generate the fixed points of the return map and then launch the system from a variety of initial conditions to obtain a rough picture of the domain of attraction of the stable manifold, $\mathcal{F} \mathcal{P}_{S}$. Figure 3 shows the results of these simulations for a hopper with leg length, $r_{t d}=.7$, spring constant $\omega_{0}^{2}=16$, and desired forward velocity $\dot{x}^{*}=1.8$. In this plot, the solid line at $\dot{x}=1.8$ represents the desired forward velocity, the thick curve represents the fixed point curve (compare Figure 3 with Figure 2) and the shaded set corresponds roughly to the set of initial conditions which get mapped to the attracting set $\mathcal{F} \mathcal{P}_{S}$. From the plot we can see that the Raibert controller provides a good region of attraction. However, since the resulting fixed point curve does not correspond exactly to the value of $\dot{x}^{*}$, the controller will regulate the forward velocity to a value near $\dot{x}^{*}$, but not necessarily equal to it.

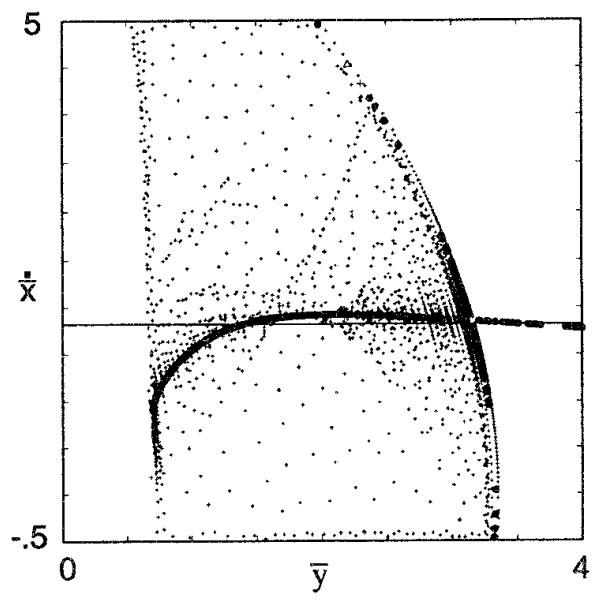

Figure 3: Region of attraction which arises from using a Raibert controller with $\dot{x}^{*}=1.8$ on a simplified hopper with $r_{t d}=.7, \omega_{o}^{2}=16$.

\subsubsection{New Control Law}

Recall that the fixed point condition is $\gamma\left(z, \theta_{t d}\right)=$ $\pi$ and a $\left(\boldsymbol{z}, \theta_{t d}\right)$ satisfying this relation results in $f_{u}\left(\boldsymbol{z}, \theta_{t d}\right)=i d$. Thus, given a state $\boldsymbol{z}_{i}$, we could numerically solve the above expression for $\theta_{t d}$ and use this value as the control input, resulting in $f_{u}=i d$. Implementing such a control law turns any point into a fixed point, $\boldsymbol{z}_{\boldsymbol{i}}=\boldsymbol{z}^{*}$. However, since we are interested in regulating about a desired value, we introduce the following proportional law

$$
p(\bar{y}, \dot{\bar{x}})=\left[\begin{array}{c}
\bar{y} \\
\dot{\bar{x}}^{*}+k\left(\dot{\bar{x}}^{*}-\dot{\bar{x}}\right)
\end{array}\right]
$$

and select the control input to be the numerical solution for $\theta_{t d}$ of the implicit fuction $\gamma\left(p(\bar{y}, \dot{x}), \theta_{t d}\right)=\pi$. This control law was implemented in simulation for a hopper with $r_{t d}=.7, \omega_{0}^{2}=16$, and $\dot{x}^{*}=1.8$ (the same values used for the simulations using the Raibert controller), resulting in the phase portrait shown in figure 4 . Comparing this with Figure 3 it is observed that this new control law, which relies more strongly on the system dynamics, gives better regulation (initial conditions actually iterate to $\dot{x}^{*}$ ) and a larger region of attraction. 


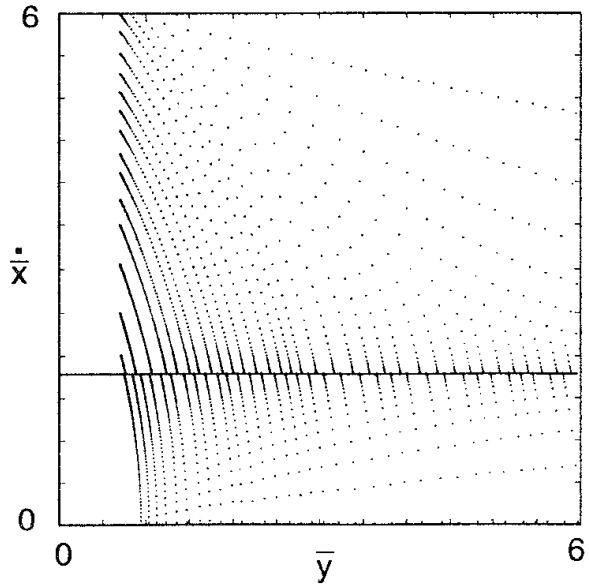

Figure 4: Region of attraction for which arises from using the new control law with $\dot{x}^{*}=1.8$ on a simplified hopper with $r_{t d}=.7, \omega_{o}^{2}=16$.

\section{Conclusion}

While a growing previous literature concerns the control of vertical hopping height for the Raibert planar hopper, no analytical work seems yet to have considered the control of forward velocity. In this paper, we have studied the planar hopper, where control is exercised solely in the forward placement of the foot at touchdown.

The major contribution of this paper is the derivation of a closed-form return map (3.2) for a simplified model of the planar hopper. This makes it possible, for the first time, to study analytically the stability of the forward velocity equilibrium behaviors.

In this work, we have introduced a feedback controller modelled on Raibert's design and characterized the fixed points of the closed loop system. We have studied the local stability of these fixed points. Simulations suggest that both $\mathcal{F} \mathcal{P}_{S}$ and its domain of attraction grow as the spring constant is increased [6]. Furthermore, while Raibert's simple decoupled feedback yields good regulation, better regulation can be achieved using coupled feedback which takes into account the dynamic structure of the robot, such as the control law of section 4.2.3. However, since we are interested in finding simple control laws, we must ask ourselves whether the more complicated control laws are worth the price - both sensing and computation are dramatically increased. We hope the analysis initiated in this paper will help us find a decoupled Raibert-like feedback which gives "global" regulation around the invariant manifold.

Thus, while local stability results are of interest, the real hope is to understand the global properties of the nonlinear closed-loop control system, $f_{u}$. As this work continues we would like to pursue stronger stability results and reintroduce the vertical component of the control.

Work in progress suggests the possibility of extending this sort of analysis to more interesting leg kinematics. Before carrying this much further, we are attempting to systematically determine the operating regimes over which the constant angular momentum assumption is valid.

\section{Acknowledgements}

We would like to thank Marc Raibert for a number of illuminating discussions bearing on this problem and John Guckenheimer for his continuing tutorial efforts on our behalf. In addition, we thank John Guckenheimer and Allen Back for helping us get up and running with dstool.

\section{References}

[1] Guckenheimer, J. and Holmes, P. 1983 Nonlinear Oscillations, Dynamical Systems, and Bifurcations of Vector Fields. New York: SpringerVerlag.

[2] Guckenheimer, J., Myers, M.R., Wicklin, F.J., and Worfolk, P.A. dstool: A Dynamical System Toolkit with an Interactive Graphical Interface. Center For Applied Mathematics, Cornell University. 1993.

[3] Koditschek, D. E., and Bühler. 1991. Analysis of a simplified hopping robot. International Journal of Robotics Research 10(6):587-605.

[4] M'Closkey, R. T., and Burdick, J. W. 1993. Periodic motions of a hopping robot with vertical and forward motion. International Journal of Robotics Research 12(3):197-218.

[5] Raibert, M. H. 1986. Legged Robots That Balance. Cambridge, MA: MIT Press.

[6] Schwind, W. J. and Koditschek, D. E. Control of Forward Velocity for a Simplified Planar Hopping Robot. Technical Report CGR-94-12, EECS Department, College of Engineering, The University of Michigan. Ann Arbor, MI. 1994.

[7] Schwind, W. J. and Yoon, A. 1993. Analysis of a Hopping Robot. Paper submitted in fulfillment of research requirement for EECS 566, The University of Michigan. Ann Arbor, MI. December, 1993.

[8] Vakakis, A. F., Burdick, J. W., and Caughy, T. K. 1991. An 'interesting' strange attractor in the dynamics of a hopping robot. International Journal of Robotics Research 10(6):606-618. 